easy to do PA with patients during their shift, while many reported they were able to encourage exercise but were unable to accompany patients to sessions. Specifically, participants reported lack of time (40\%), high level of clinical activity (32\%), lack of staff (30\%), lack of PA resources inside the wards $(20 \%)$ and conflicting priorities (18\%), stopping them from helping patients to do more exercise. However, they felt more staff (28\%), time dedicated to PA (26\%), on-ward resources (18\%), access to the gym and gardens (18\%), staff dedicated to PA (16\%) and staff trained in facilitating PA (10\%), would help participants promote PA on the ward. Other suggestions to enable PA included a change in ward culture, valuing and promoting PA, daily patient encouragement by all MDT members instead of only occupational therapists, and PA promotion as part of mental health treatment and as physical health strategy. Finally, $70 \%$ of participants said they exercised regularly, although some reported lack of time or motivation, work commitments and workload-related exhaustion reducing their ability to exercise.

Conclusion. Participants acknowledged the importance of PA for physical and mental health. Furthermore, they described multiple enablers and barriers. Prioritising PA during admission, providing on-ward activities, educating/training staff, reiterating that PA promotion is within all MDT members' job roles, and offering organisational support can contribute to improved PA provision and regular involvement of patients.An integrative approach to mental health and wellbeing, promoting PA in inpatient psychiatric settings is required.

\section{Improving accessebility to psychiatry in NHS Tayside}

Thomas Leung*, Lori-an Etherington and Neil Stevenson

NHS Tayside

${ }^{*}$ Corresponding author.

doi: 10.1192/bjo.2021.546

Aims. Our aim is to improve the accessibility of Psychiatry to other specialties when being contacted for review and advice, both in hours and out of hours.

Background. From clinical contact and informal conversations, other specialties sometimes have difficulties contacting psychiatry for advice/review. The aim of this is quality improvement project is to determine how accessible we are to other specialties and work on improving how we communicate with the general hospital. Method. We created a questionnaire for colleagues from other specialties to fill in from 26/9/19 for 6 weeks. We gathered information regarding their grade, work site, previous contact with psychiatry, whether they knew where to find our contact information and if they could identify the correct method to ask for advice from general adult psychiatry (GAP), Psychiatry of old age (POA), and out of hours psychiatry $(\mathrm{OOH})$. We also asked colleagues to put in free text comments regarding their experience in contacting psychiatry. We also asked if our colleagues were aware of how to perform an Emergency Detention Certificate as this is advice we sometimes give which does not always need our input immediately.

Result. There was a total of 39 responses, 29 from Ninewells Hospital (NW) and 10 from Perth Royal Infirmary (PRI). There was a mixture of staff grades from Foundation Doctors to Consultants. 23/39 colleagues knew where to find contact information for Psychiatry, 14/39 colleagues correctly answered how to contact GAP (Phone), 15/39 colleagues correctly answered how to contact POA (Email), 15/39 colleagues correctly identified who to contact $\mathrm{OOH}$, and $16 / 34$ colleagues who could do emergency detentions (FY2+) knew how to do one. Free text comments often referred back to the difficulty of finding the right grade of staff first try, Feedback from PRI where there was no dedicated Liaison Service and relies on a duty doctor system was less positive, with terms 'tricky', 'difficulty', 'awkward' used in majority of responses.

Conclusion. From our results we can conclude that contacting Psychiatry in NHS Tayside can be confusing for other specialties. Taking this forward, we will utilize the 'referral finder' system in NHS Tayside and review the existing information available, and to update the contact information for our subspecialties to make contact ourselves more streamlined and accessible. We will also review appropriate clinical protocols that we can link to our page on referral finder to help save time for our colleagues as well.

\section{Improving the physical healthcare of COVID-19 patients in inpatient psychiatric settings}

Marissa Lewis*, Karolos Dionelis, Miguel Vecida, Rebecca Phelps, Helen Hopwood, Lawrence Yong, Jason Ng, Sara Veeramah, Miranda Lloyd and Tom Clark

Oxleas NHS Foundation Trust

${ }^{\star}$ Corresponding author.

doi: 10.1192/bjo.2021.547

Aims. COVID-19 can spread rapidly in psychiatric inpatient settings. Previous studies have found that patients have a higher risk of hospitalisation and death than adults in the community. The aim of this project was to improve the care of patients with COVID-19 in psychiatric inpatient settings.

Method. A baseline audit was conducted of care COVID-19 patients received in wards that experienced outbreaks in January 2021 in a London Mental Health Trust. Clinical notes were reviewed for management plans, including clear documentation of risk of serious illness, frequency of vitals monitoring, and thresholds for escalation to medical teams.

A new protocol was subsequently developed and implemented at one inpatient unit: "COVID-19: Early Identification of Risk and Management". This included an adjusted 4C mortality score to determine risk of deterioration, and schedules for observation monitoring based on this outcome. Each schedule specified separate frequencies of monitoring of critical observations (oxygen saturations, respiratory rate) and routine observations, thus minimising unnecessary staff exposure. It prompted venous thromboembolism (VTE) assessment and documentation of escalation criteria.

Result. 44 patients were identified across three working age (WAA, $\mathrm{n}=29)$ and two older age $(\mathrm{OA}, \mathrm{n}=15)$ adult wards. $7.5 \%$ of WAA and $33.3 \%$ of OA patients were hospitalised. $20 \%$ of OA patients died following a positive test. $58 \%$ of patients had a documented management plan for COVID-19, but only $56 \%$ mentioned observation frequency, 19\% escalation criteria, and $9 \%$ risk of serious disease. No patient received a repeat VTE assessment following diagnosis. The audit identified inconsistent approaches to COVID-19 management between wards, and found no relationship between risk of deterioration and frequency of observation monitoring. Following implementation of this protocol, $100 \%(n=4)$ of patients had a robust plan for COVID-19 management, and 100\% received a VTE assessment. Conclusion. The audit supported previous findings that psychiatric inpatients are at risk of serious COVID-19 infection. This highlights an urgent clinical and ethical need to optimise COVID-19 care in psychiatric inpatient settings. The results of this audit suggest that risk factors for severe infection and elements of routine care are not widely understood or implemented by clinical staff. Introducing evidence-based protocols to support clinicians in managing the physical healthcare of these patients 
may be one way of promoting best practice. The improvement in care observed in the pilot study has resulted in this protocol being rolled out across the Trust in an ongoing quality improvement project.

Improving the quality of GP referrals to the Croydon Assessment \& Liaison Team

Gabriella Lewis*, Lucia Chaplin, Gareth Knott, Alexandra Coull and Lamide Sobamowo

South London and Maudsley NHS Foundation Trust

${ }^{\star}$ Corresponding author.

doi: 10.1192/bjo.2021.548

Aims. To increase the percentage of GP referrals to the Croydon Assessment \& Liaison (A\&L) Team deemed to be of 'good quality'. The A\&L Team receives a large number of referrals daily from GPs, and it was identified that many of these referrals did not include important and relevant information, leading to delays in patient assessments.

Method. A questionnaire was distributed to A\&L MDT members to collect information about what information they consider important in a GP referral. The project team reviewed the results of the questionnaire, along with current policies and guidelines, to create a set of criteria by which to assess the quality of GP referrals, as there was no pre-existing gold standard available. A random sample of 6 GP referrals per week stratified by locality was collected and assessed against these criteria.

Using Plan-Do-Study-Act (PDSA) methodology change ideas were generated, and a GP referral form was identified as an important intervention to adopt. A previously-developed draft form was updated after a round of consultations with various stakeholders including Assessment \& Liaison staff, GPs and the CCG. The new GP referral form was uploaded to the GP DSX electronic referrals platform and GP practices were also emailed directly to encourage them to use the new form.

The proportion of GP referrals deemed to be of good quality was compared pre and post-intervention. Uptake of the new GP referral form was recorded as a process measure, and the length of time taken to discuss referrals at A\&L daily referrals meetings as a counterbalance measure.

Result. At baseline 33\% of GP referrals were deemed to be of good quality using the developed criteria. This improved to $58 \%$ after implementation of the new referral form in January 2021. There was poor overall uptake of the form, with only $32.5 \%$ of GP referrals utilising the new form so far, however of the referrals received on the new form $69 \%$ fulfilled the criteria for good quality. Comparison of length of discussion required for referrals with and without the new form showed no significant difference (7.7 and 7.6 minutes respectively).

Conclusion. Implementation of a standardised GP referral form was effective at increasing the proportion of referrals deemed to be of good quality. However, further PDSA cycles focused on improving uptake of the form will be required.

A community service review of the quality of inpatient discharge summaries from six inpatient wards at $\mathrm{St}$ Charles Hospital: an initial audit and quality improvement recommendations

Omar Mahmoud ${ }^{1 \star}$, Jasna Munjiza ${ }^{2}$ and Jacob King ${ }^{3}$

${ }^{1}$ ST5 General Adult psychiatry Trainee, Pall Mall, North Kensington and Chelsea CMHT; ${ }^{2}$ Consultant General Adult psychiatrist, Pall
Mall, North Kensington and Chelsea CMHT and ${ }^{3}$ Core trainee, Pall Mall, North Kensington and Chelsea CMHT ${ }^{\star}$ Corresponding author.

doi: $10.1192 /$ bjo.2021.549

Aims. To discuss whether Discharge summaries include important information to community mental health teams .

To identify patterns and produce recommendations for change by Quality improvement methods .

Method. A convenience sample was selected of the first 5 patient discharges from each of the 6 adult inpatient wards at St Charles Hospital. This represented a total of 30 reviewed summaries. Outcome items were generated following discussion with community psychiatric colleagues based on those aspects of an admission thought to be of most use to a community mental health team. These were; reason for admission, diagnosis, circumstances of admission, progress on the ward, risk assessment, physical health, legal status on discharge, discharge medication, discharge management plan, contact details. Basic identification was also recorded as was the ward and date of discharge

Result.

- Only 3.3\% (1/30) of discharge summaries were complete of all items.

- However 23.3\% (7/30) were almost complete, failing to record only a single item, and a further 2 missing only 2 of 10 items. There was a bimodal distribution (Graph 1).

- Seven (7/30) discharge summaries provided no information. Of these, four (4/7) discharge summaries were written in the progress notes directly, rather than using the discharge summary proforma.

- The 'reason for admission' item was a clear low outlier with only $2 / 30$ reporting this piece of information. For a number of cases, this was recorded unhelpfully as "in crisis".

Conclusion. There was limited evidence of systemic patterns, however some wards showed internal stark differences with some summaries complete or almost complete and others empty.

The key findings from this report are the high number of discharge summaries which have either no responses to them (7/30). This may indicate that the writer did not know how to use the current discharge template, and therefore support with using this is indicated. For those with a very low (7/30) number of item responses, we might conclude that these discharge summaries were written by someone with knowledge of using the system, but for another reason did not complete the majority of the items asked, and for this reasons are not immediately clear. Similarly, as highlighted above the main low outlying result relates to the apparent widespread practise of writing "in crisis" as the "reason for admission', unfortunately to community teams this is an unhelpful and self-evident response.

Innovating in CMHT's: mental health wellbeing group visits

Samuel Mammolotti Parkinson*, Ismail Laher and Shola Johnson

Leeds \& York Partnership NHS Foundation Trust

${ }^{\star}$ Corresponding author.

doi: 10.1192/bjo.2021.550

Aims. 'Group consultations/visits' are described as providing shared medical appointments delivering a range of care options and education by clinicians while providing elements of patient choice, empowerment and peer support. 